\title{
Der Jungle als Stadt
}

Rezension zu Michel Agier / Yasmine Bouagga / Mael Galisson / Cyrille Hanappe / Mathilde Pette / Philippe Wannesson (2020): Der „Dschungel von Calais“. Über das Leben in einem Flüchtlingslager. Bielefeld: transcript.

Abb. 1 Titel des Buches (transcript Verlag)

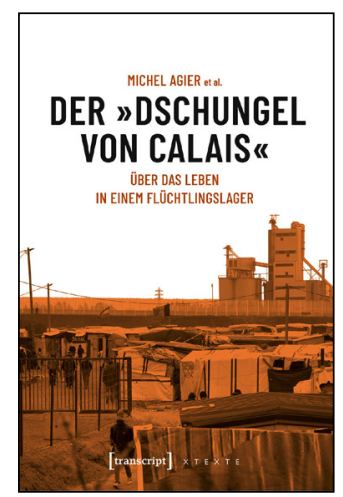

,Calais` steht für weit mehr als eine Stadt im Norden Frankreichs. Die Lage am Ärmelkanal macht Calais für Logistik und Reiseverkehr zwischen dem europäischen Festland und Großbritannien relevant. Aber nicht alle Reisenden sind erwünscht. Calais wurde zum zentralen Ort eines restriktiven Grenzregimes, gekennzeichnet durch kilometerlange Zäune, Polizeikontrollen und Stacheldraht. Jedoch greift ein alleiniger Fokus auf Restriktion zu kurz, um diesen Ort in seiner Gänze zu verstehen. Denn Calais steht auch für migrantisches Leben und Selbstorganisation, sichtbar in einer Vielzahl von „makeshift camps“ (provisorischen Lagern, Katz 2017) - Calais steht für ,den Dschungel' beziehungsweise für eine Vielzahl von Jungles[1], wie sich später noch zeigen wird.

Das von dem französischen Anthropologen Michel Agier, der Soziologin Yasmine Bouagga und vier weiteren Autor*innen veröffentlichte Buch Der „Dschungel von Calais.“ Über das Leben in einem Flüchtlingslager (Agier et al. 2020) leistet einen wichtigen Beitrag, diesen Ort besser zu verstehen. Bisher stand Calais kaum im Fokus deutschsprachiger Debatten zu Migration und Grenzregimen. Wenn Calais hier in den Medien thematisiert wurde, war dies meist mit verkürzten Analysen und Klischees über Chaos und Gewalt verbunden. Ausnahmen stellen unter anderem die Texte von Thomas Müller dar (z. B. Müller/Schlüper 2018; Müller/Zinflou 2018), der auch das Vorwort zur deutschen Ausgabe dieses Buches geschrieben hat (Müller 2020). Wie er dort schreibt, lag der Fokus deutschsprachiger Debatten um 2015 vor allem auf der Balkanregion und verschob sich in den vergangenen Jahren auf die Ereignisse im Mittelmeer. Calais blieb ein „Randthema“ (ebd.: 19). Ein Blick auf Calais ist jedoch wichtig, denn er eröffnet neue Perspektiven: für Fragen der Migrationsforschung, aber auch der (kritischen) Stadtforschung.

Michel Agier leitete das Buchprojekt, zu dem eine Reihe von weiteren Autor*innen beigetragen haben: Im Buch versammeln sich Beiträge aus der Soziologie, Anthropologie und Architektur, aber auch von zivilgesellschaftlich involvierten Personen. Diese Sammlung an Perspektiven macht das Buch spannend und auch die Kapitel spiegeln die Breite der Analyse 
wider: So geht es um die historische Entwicklung der Jungles, eine Analyse der Unterbringungsarchitekturen, Berichte über das Alltagsleben, aber auch um Solidarität und Unterstützung und den Prozess der Räumung des Jungles 2016. Das Buch fokussiert den medial bekanntesten Jungle, welcher 2015 und 2016 existierte. Im Folgenden werde ich mich mit dem Jungle im Singular vor allem auf diesen Ort beziehen. Die Autor*innen schaffen es jedoch, diesen Ort nicht zu essentialisieren, sondern in einen breiteren zeitlichen (1986-2016) und räumlichen Rahmen (mit Verweise auf Lager in Dünkirchen und Paris) einzubetten. Es geht also nicht um den Jungle, sondern immer auch um die Jungles von Calais.

Vor allem die Detailliertheit der Beiträge ist bemerkenswert. Den methodischen Ansatz des Forschungsprojektes beschreiben Agier et al. mit dem Begriff „forensic anthropology“ als „kollektives anthropologisches Gutachten“ (S. 45). In diesem Sinne und durch die detaillierten Einblicke schafft das Buch eine umfassende Analyse der Jungles, die eine zu simple Romantisierung vermeidet. Die Jungles sind Ortevon Rassismus, Prekarität und Repression, aber gleichzeitig auch Orte von Solidarität und Autonomie. Das Buch wurde 2018 auf Französisch veröffentlicht. Da die deutsche Übersetzung von Wolfgang Freund erst 2020 erschien und sich die Situation von Migrant*innen in Calais im ständigen Wandel befindet, stellt das Vorwort von Thomas Müller eine wichtige Ergänzung, Einordnung und Aktualisierung dar.

Eine zentrale These des Buches ist, dass der Jungle eine urbane Struktur angenommen hat und zu einer „Stadt der Migrant_Innen“ geworden ist (S. 112). Das ist für die kritische Stadtforschung die wohl relevanteste Aussage des Buches und damit steht sie auch im Fokus dieser Rezension. In Calais schufen Migrant*innen einen Ort, an dem bis zu 10.00o Menschen lebten. Der Jungle war geprägt von einer zentralen Straße mit Infrastruktur und Läden, Restaurants, Schulen, Kirchen und Moscheen. Neben einer genauen Beschreibung des Ortes werden im Buch auch die komplexen sozialen Beziehungen an diesem Ort analysiert und der entstehende kosmopolitische und politische Charakter des Jungles betont. Die Autor*innen nutzen dafür häufig den Begriff Bidonville. Dieser Begriff bezeichnet in der frankophonen Welt vor allem ,Slums' und informelle Siedlungen etwa in ehemaligen französischen Kolonien wie Tunesien und Algerien. ,Bidonville“ wird dabei häufig mit Chaos und Defiziten der Stadtentwicklung verbunden. In diesem Buch wird der Begriff, Bidonville‘ jedoch positiv besetzt. Es geht darum, eine Stadt im Entstehen, im Übergang vom Lager (camp) zur Stadt (cité) zu beschreiben, wie Müller im Vorwort erläutert (Müller 2020: 22). Mit Blick auf die Räumung des Jungles im Herbst 2016 schreiben Agier et al.:

„Mit dem Bidonville erfanden sich die Migrant_Innen in Frankreich jene gastvolle Stadt selbst, die die Regierung ihnen verweigerte. Genau das war es, worauf der Staat schließlich reagierte: gegen das Camp, das nach und nach aus dem Schatten trat und zu sichtbar, zu autonom und zu politisch wurde.“ (S. 194)

Die Autor*innen schaffen es mit dieser These, den Blick auf Calais zu drehen. Der Jungle ist nicht der chaotische und ,gescheiterte ' Ort, wie er in den Medien häufig dargestellt wurde. Es ist ein Ort, geprägt von einer komplexen und kosmopolitischen Urbanität, der von Handlungsmacht und 
Emanzipation zeugt. Dies ist eine spannende These, welche die Grenzen zwischen räumlichen Anordnungen von Stadt/Lager ins Wanken bringt. Es geht nicht um zwei unvereinbare Gegensätze, sondern darum, dass Lager zu Städten werden können. Der Ansatz baut auf früheren Studien von Agier auf, in denen er die Urbanisierung von Migrant*innenlagern im globalen Süden als „city-camps“ beschreibt (Agier 2002, 2011) - eine Dynamik, die auch von weiteren Wissenschaftler*innen wie dem Architekten Manuel Herz (2013) analysiert wird.

Diese These ist nicht nur für die Migrationsforschung und Debatten zur Lager-Unterbringung von Bedeutung. Auch für die (kritische) Stadtforschung bietet sie Anknüpfungspunkte, um bestehende Ansätze und Konzepte, etwa zum Verständnis von Stadt und Urbanisierung, zu hinterfragen. So spannend das für weitere Debatten sein kann - das Buch wirft die These der Urbanisierung leider meist nur auf, ohne sie tiefergehend zu entwickeln. An mehreren Stellen finden sich Überschriften und Sätze wie „die Kunst Städte zu bauen“ (S. 101), „eine Stadt wurde erschaffen“ (S. 135) oder „kosmopolitische Schnittstelle der Solidaritäten“ (S. 139), aber eine weiterführende (theoretische) Auseinandersetzung bleibt aus. Es bleibt häufig unklar, was diese Sätze bedeuten könnten. Auch findet sich nirgends eine klare Definition von Stadt und dem Urbanen. Dies zeigt sich auch in der Verwendung des Begriffes ,Bidonville‘, der von den Autor*innen nicht an existierende Debatten zurückgebunden wird. So verbindet das Buch ,Bidonville ' mit der positiven Entwicklung einer Urbanisierung, jedoch ohne kritisch auf die häufig negativen Beschreibungen von Bidonvilles einzugehen. Die These der Urbanisierung des Jungles bleibt damit fragil. Das reichhaltige empirische Material hätte dafür stärker in eine theoretisch untermauerte These überführt werden können. Das Buch öffnet zwar Perspektiven für eine Diskussion, an einigen Stellen hätte ich mir aber ein wenig mehr Theorie und Bezug auf bestehende Debatten gewünscht.

So hätte etwa die Beziehung von Stadt und Lager weiter entwickelt werden können. Bezogen auf die früheren Werke von Agier und seine These einer Urbanisierung von Lagern finden sich auch kritische Stimmen (z. B. Malkki 2002). Lucas Oesch (2020) schreibt, dass es weniger um die Frage geht, ob Lager Städte sind, sondern auch um die Beziehung zwischen Lager und (umliegender) Stadt. Diesen Forschungsansatz verfolgt zum Beispiel auch Romola Sanyal (2014), und er ist für die Stadtforschung von Interesse. So findet sich auf dem Titelbild des Buches ein Foto des Jungles. Im Hintergrund sind Zäune und ein Industriegebiet der Stadt Calais zu sehen. Neben der Frage, ob und wie der Jungle zu einer Stadt geworden ist, wäre hier auch ein Blick auf die bestehenden Beziehungen zwischen der Stadt Calais und dem Jungle von Calais interessant gewesen. Inwieweit war der Jungle auch Teil der Stadt Calais? Was sagt die Lage im direkten Umfeld eines Industriegebietes über diese Beziehung aus? Solch eine Analyse hätte die These der Urbanisierung des Jungles vertiefen können, denn der Prozess einer Urbanisierung findet in der Peripherie einer bestehenden Stadt statt.

Jenseits der Frage der Urbanisierung wäre ein Fokus auf die Beziehungen zwischen Jungle und Calais auch an weiteren Stellen spannend gewesen. So könnte etwa betrachtet werden, wie sich Calais als Zentrum eines Grenzregimes mit einem logistischen Knotenpunkt überschneidet, durch 
den Eurotunnel, aber auch durch den großen Fährhafen als Verbindung zwischen EU-Festland und Großbritannien. Wie wird hier versucht, die Bewegung von Migrant*innen auf der einen Seite zu verlangsamen, zu steuern und zu selektieren, und auf der anderen Seite, den Warenverkehr zu beschleunigen? Wie werden logistische Abläufe gleichzeitig gestört? Wie verlangsamt Calais als Grenzregime den Warenverkehr, indem Migrant*innen Lastwagen aufhalten, um nach Großbritannien zu kommen, aber auch indem aufwendige Polizeikontrollen stattfinden, welche genau diese Aktionen von Migrant*innen verhindern sollen? Solche Fragen hätten zum einen die Komplexität des Ortes weiter beleuchten, zum anderen aber die Beschreibung des Jungles auch mit weiteren (theoretischen) Debatten wie etwa zu Mobilität, Grenzregimen und Logistik verknüpfen können.

Bezogen auf den methodischen Ansatz eines „(kollektiven) anthropologischen Gutachtens“ (S. 45) ist es vielleicht zu viel erwartet, tiefergehende theoretische Debatten und Antworten auf all diese Fragen zu erwarten. Die aufgeworfenen Themen zeugen daher auch von der Neugier, welche das Buch in mir geweckt hat. Denn was das Buch durch seinen Ansatz leistet, ist eine sehr detailreiche Darstellung der Jungles von Calais. Es bildet eine Grundlage für weitere Forschung, um Fragen zu entwickeln und in theoretisch untermauerte Analysen zu überführen. Bei dem vorliegenden methodischen Vorgehen überrascht es allerdings, dass in dem Buch nur wenig Reflexion und Details über Methodologie zu finden sind, zum Beispiel dazu, wie genau vor Ort vorgegangen wurde, welche Methoden angewandt wurden und welche Herausforderungen aufkamen. Und obwohl eine Vielzahl von Personen aus Wissenschaft und Zivilgesellschaft das Buch geschrieben haben, sind Stimmen von Migrant*innen wenig zu vernehmen. Die Kapitel berichten zwar über Blickwinkel und den Lebensalltag von Migrant*innen, es ist aber kein expliziter Fokus des Buches, diese in allen Kapiteln selbst zu Wort kommen zu lassen. Andere Buchprojekte, wie Voices from the, Jungle (Calais Writers 2017), haben genau das in den Vordergrund gerückt, indem Migrant*innen des Jungles zu den Autor*innen wurden. Auch wenn Agier et al. diesen Ansatz nicht per se wiederholen müssen, so wäre zumindest eine Reflexion und Begründung dieser Entscheidung und des methodisches Vorgehens insgesamt wichtig gewesen.

Trotz dieser Kritikpunkte stellt das Buch einen wichtigen Beitrag dar, um den Jungle beziehungsweise die Jungles von Calais besser verstehen zu können. Gerade für die deutschsprachige Debatte schließt das Buch Lücken. Es bietet einen detaillierten Blick auf den Jungle abseits von Stereotypen. Es räumt auf mit gängigen Klischees über den Jungle als Ort von Chaos und Gewalt. Auch wenn das Buch mit der Räumung des Jungles 2016 schließt, zeigen das Entstehen von neuen Lagern, erneute Räumungen (wie etwa im Juli 2020) und die starke Zunahme von Seepassagen per Boot, dass es an Aktualität nicht fehlt.[2] Im August 2020 kündigte der britische Premierminister Boris Johnson an, Marineboote vor der Küste Frankreichs einzusetzen, um diese Überfahrten zu verhindern. Calais bleibt somit ein zentraler Ort des europäischen Grenzregimes, aber gleichzeitig auch ein Ort von migrantischer Autonomie. Um diesen Ort abseits von klischeebeladenen Mediendiskursen zu verstehen, lohnt sich ein Blick in das Buch - auch wenn weitere (theoretische) Überlegungen uns überlassen sind. 


\section{Endnoten}

[1] Wie Thomas Müller im Vorwort des Buches anmerkt, ist die deutsche Übersetzung des englischen und französischen Begriffs ,jungle problematisch. Die Bezeichnung jungle entstand in den 200oer Jahren in Anlehnung an ein Lager in einem Waldgebiet bei Calais, welches von Migrant*innen mit dem paschtunischen Wort dzangal (Wald) bezeichnet wurde. Seit diesem Zeitpunkt verwenden Migrant*innen und Unterstützungsstrukturen vor allem den Begriff jungle, um die provisorischen Lager in Calais zu beschreiben. In der deutschen Übersetzung geht dieser Kontext jedoch verloren und ,der Dschungel' ist verbunden mit einer medialen Repräsentation dieser Orte, welche koloniale Stigmata von Chaos und Exotisierung reproduziert. Im Buch wird daher vor allem der Begriff ,Jungle‘ (und ,Jungles“ im Plural) verwendet. Dem möchte ich auch in dieser Rezension folgen.

[2] Der Blog calais.bordermonitoring.eu wurde im Zuge der Corona-Pandemie ins Leben gerufen und bietet einen guten Überblick über die aktuellen Ereignisse in Calais.

\section{Autor_innen}

Christian Sowa arbeitet zu Fragen von kritischer Stadt- und Migrationsforschung. In seiner Promotion befasst er sich mit der Unterbringung Geflüchteter in Berlin.

christian_sowa@soas.ac.uk

\section{Literatur}

Agier, Michel (2002): Between War and City: Towards an Urban Anthropology of Refugee Camps. In: Ethnography 3/3, 317-341.

Agier, Michael (2011): Managing the undesirables. Refugee Camps and Humanitarian Government. Cambridge: Malden.

Agier, Michel / Bouagga, Yasmine / Galisson, Mael / Hanappe, Cyrille / Pette, Mathilde / Wannesson, Philippe (Hg.) (2020): Der „Dschungel von Calais“. Über das Leben in einem Flüchtlingslager. Bielefeld: transcript.

Calais Writers (2017): Voices from the „Jungle“. Stories from the Calais Refugee Camp. London: Pluto Press.

Herz, Manuel (Hg.) (2013): From Camp to City: Refugee Camps of the Western Sahara. Baden/London: Lars Müller.

Katz, Irit (2017): Between Bare Life and Everyday Life: Spatializing Europe's Migrant Camps. In: Architecture_MPS 12/2, 1-21.

Malkki, Liisa (2002): News From Nowhere: Mass Displacement and Globalized „Problems of Organization“. In: Ethnography 3/3, 351-36o.

Müller, Thomas (2020): Eine Vorbemerkung zur deutschsprachigen Ausgabe. Die Aktualität des Jungle. In: Michel Agier / Yasmine Bouagga / Mael Galisson / Cyrille Hanappe / Mathilde Pette / Philippe Wannesson (Hg.), Der „Dschungel von Calais“. Über das Leben in einem Flüchtlingslager. Bielefeld: transcript, 9-35.

Müller, Thomas / Schlüper, Uwe (2018): Dynamiken des Jungles. Calais und das europäischbritische Grenzregime. München: bordermonitoring.eu.

Müller, Thomas / Zinflou, Sascha (2018): Die Urbanität des Jungle. Calais und die Möglichkeiten einer migrantischen Stadt. In: movements 4/2, 129-160.

Oesch, Lucas (2020): An improvised dispositif: The invisible urban planning in the refugee camp. In: International Journal of Urban and Regional Research 44/2, 349-365.

Sanyal, Romola (2014): Urbanizing Refuge: Interrogating Spaces of Displacement. In: International Journal of Urban and Regional Research 38/2, 558-572. 